This item was submitted to Loughborough's Research Repository by the author.

Items in Figshare are protected by copyright, with all rights reserved, unless otherwise indicated.

\title{
Modelling and simulation of functional product system availability and support costs
}

\author{
PLEASE CITE THE PUBLISHED VERSION \\ PUBLISHER \\ (C) Inderscience
}

VERSION

AM (Accepted Manuscript)

LICENCE

CC BY-NC-ND 4.0

\section{REPOSITORY RECORD}

Lofstrand, Magnus, Sean Reed, Magnus Karlberg, J.D. Andrews, Lennart Karlsson, and Sarah J. Dunnett. 2012. "Modelling and Simulation of Functional Product System Availability and Support Costs". figshare. https://hdl.handle.net/2134/11254. 
This item was submitted to Loughborough's Institutional Repository (https://dspace.lboro.ac.uk/) by the author and is made available under the following Creative Commons Licence conditions.

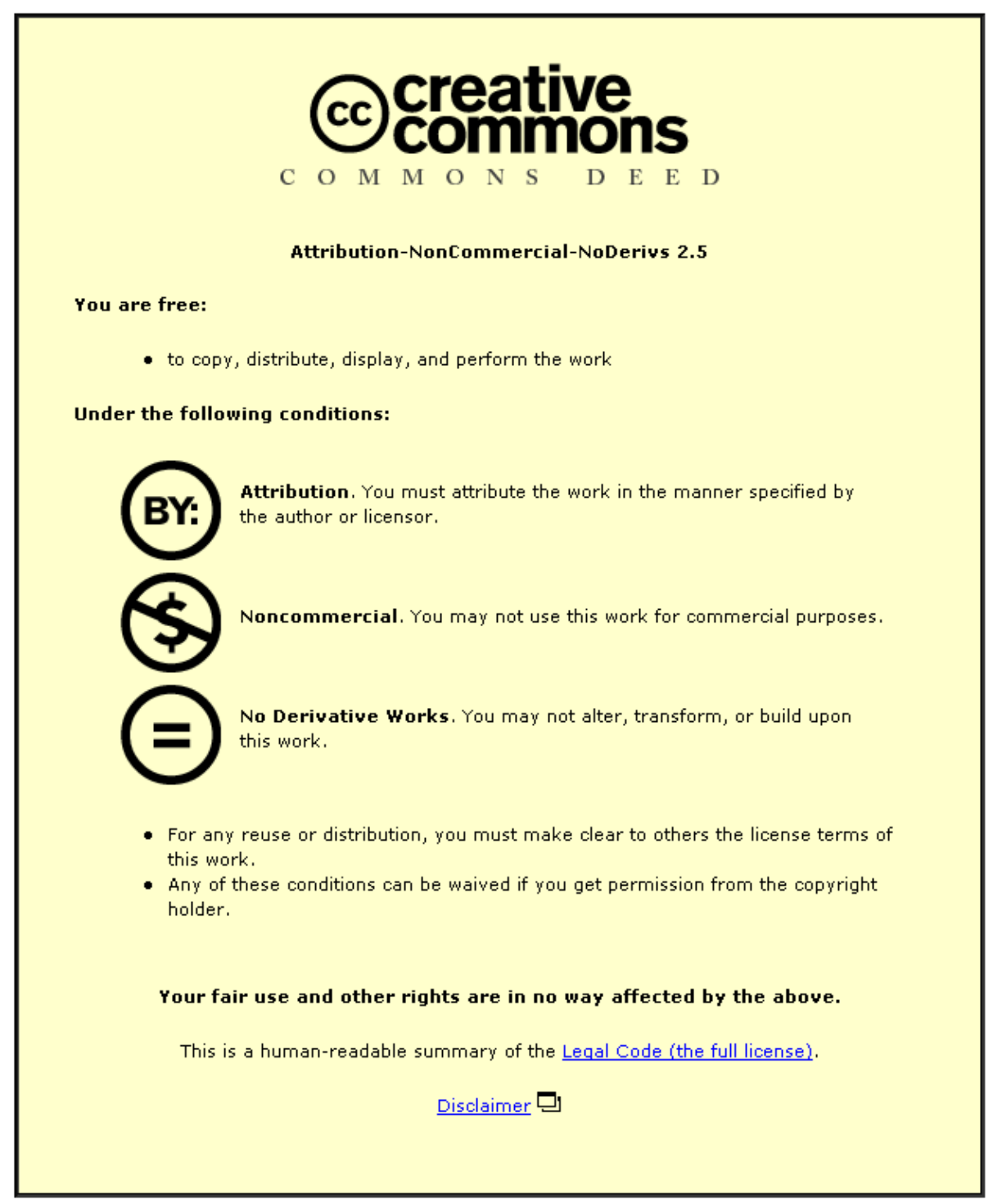

For the full text of this licence, please go to: http://creativecommons.org/licenses/by-nc-nd/2.5/ 


\title{
Modelling and Simulation of Functional Product System Availability and Support Costs
}

\begin{abstract}
Functional products (FP), total offers or product service systems, that comprise of both hardware (HW) and support services (SS) sold as an integrated offering under an availability guarantee, are becoming increasing popular in industry. This paper addresses, through modelling and simulation, the challenge faced by suppliers in developing an integrated HW and SS design to produce an FP which meets contracted availability. A recently published framework specified how an integrated model hardware and service support system model could be used to obtain functional availability predictions and perform simulation driven functional product development. This paper presents the first example of an integrated functional product model. It uses fault tree, Petri net and discrete event simulation techniques to enable the prediction of functional product availability and support costs. Such predictions are used here to evaluate and compare different service support system designs.
\end{abstract}

Keywords: Functional Product Development, Product-Service-System, service support system, discrete event simulation, Petri nets, Availability.

\section{Introduction and functional product background}

Traditionally, high-tech hardware designs have been sold as standalone products and the customer then obtains maintenance support through in-house provision or, alternatively, by purchasing it from the original equipment manufacturers (OEM) or third parties. Since the availability (J. D. Andrews \& Moss 2002) of the hardware function is often critical to the operation of the customer's business, obtaining adequate maintenance support, in the form of services and spare parts, is essential. However, these forms of arrangement are not ideal for either the customer or hardware supplier. From the customer's viewpoint, they are concerned only with obtaining availability of the hardware function at an acceptable overall cost that includes both the initial hardware purchase and the ongoing maintenance support provision. Under these arrangements, they are exposed to significant business risk due to the uncertain hardware reliability and maintenance outcomes of hightech products. This uncertainty is greatest for new product designs or older designs utilised in new applications. The result is risk of poor functional availability and high support costs which, together with the large upfront purchase price, leads to reluctance to purchase the latest hardware products despite them offering improved performance. If reliability performance turns out to be poorer than expected, the costs of (a) lost business revenue due to deficient hardware functional availability and (b) maintenance support can have a significant adverse impact upon the customer. Additionally, new hardware designs imply that the customer and third parties will lack familiarity with the products and therefore find providing adequate maintenance support difficult. However, purchasing maintenance support from the OEM results in misaligned motivations as the provider of maintenance support obtains increased revenue from higher maintenance servicing and spares costs and does not suffer directly if hardware functionality is lost. From the manufacturer's viewpoint, the traditional arrangements are also unsatisfactory, partly 
Author

because businesses are naturally risk-averse and the aforementioned risks for the customer therefore results in reduced sales. Customers are incentivised to continue using older product designs, as the build-up of experience and certainty in functional availability and support costs outweigh the potentially improved but uncertain performance (in terms of functionality, functional availability and support costs) of newer designs. Other advantages for manufacturers are that functional products provide an ongoing source of revenue, result in a closer relationship with customers, present opportunities for cost savings (e.g. use of remanufactured hardware and sharing of spare part pools between customers) and facilitate the collection of product operational and maintenance data for use in product development.

These problems have led to an increased desire and popularity for high-tech hardware to be leased by OEMs to customers as integrated packages that include a service support system (containing maintenance services amongst others) under a contract that guarantees a specified functional availability. These product sales are known under various names such as functional products or performance-based contracts (Kim et al. 2007) and can be seen as a development of product service systems (PSS) that originated in Scandinavia in the 1990's (Baines et al. 2007). These integrated products solve the set of problems inherent to hardware sold under traditional contracts that were mentioned earlier. Additionally, they lead to better product designs since the motivation for high functional availability and low support costs resides with the manufacturer. However, for the manufacturer, they also create new demands for product development (Brännström et al. 2001) and pose the potential for increased risk.

Products to be sold under functional product contracts should be developed such that the hardware and maintenance support services are devised congruently to arrive at a design with the best overall performance. This necessitates an increased focus on the design of the service support system and how it integrates with the hardware to deliver high functional availability at acceptable support costs. The supplier also now bears the costs for failing to achieve guaranteed levels of hardware function availability and of providing service support once the product is sold. For both driving the design and reducing aftersales risk, the supplier must therefore be able to predict functional availability and service support costs during the product design stage. Since the manufacturer has intricate knowledge of the product design, the potential for doing so exists. Unfortunately, whilst methods for the modelling and prediction of hardware availability have been well researched and are widely used, those for integrated hardware and service support system products are lacking.

The key contribution from this paper is a description of a modelling methodology and implementation that can provide a functional availability and support cost prediction capability to functional product manufacturers. As such, it provides a means by which they can evaluate functional product designs in terms of these metrics. It also represents the first step towards a future complete implementation of a framework that will enable simulation driven development of functional products.

The remainder of this paper is structured as follows: section 2 contains a literature review of related research, section 3 describes the functional product modelling strategy, section 


\section{Modelling and Simulation of Functional Product System Availability}

4 provides verification of the modelling through application to a simple example functional product and section 5 summarises and concludes the presented research.

\section{Literature Review}

Due to an increasing desire from manufacturers to offer functional products, researchers have begun to investigate the unique product development issues they pose and how they might be solved. Alonso-Rasgado et al (2004) researched the design issues unique to functional products and, in particular, of the service support system. One of their conclusions was that hardware reliability is well developed compared with service reliability. In a review of PSS research, Baines et al (2007) concluded that tools need to be developed which enable modelling of PSS, particularly quantitative methods. They noted that manufacturers who see the provision of services as key to their future need to be able to configure their products, technologies, operations, and supply chain to support this value offering; yet little guidance is available for manufacturers in this situation. These findings support our own experience with a number of industrial partners, who are keen to offer functional products but lack the decision support tools necessary for their design, development and sale.

Since functional products are an integrated package of hardware and service support system, both of these elements need to be included in a functional product model. As was concluded by Alonso-Rasgado et al (2004), the modelling and quantification of hardware reliability has received a huge amount of research. For a review of the state-of-the-art methods for system reliability assessment see Andrews (2009). Li and Thompson (2007) presented the first investigation into the performance of services in the context of functional products where they modelled a service as a schedule of tasks and used simulation to evaluate performance time and reliability. Reed et al (2010) developed an improved methodology for modelling the performance of a functional product service support system, where maintenance activities were again modelled as a schedule of tasks but with increased flexibility in task prerequisite constraints and the inclusion of maintenance resource constraints. Löfstrand et al (2011) published a framework (see Figure 1) that demonstrated how the design of a functional product could be driven through simulation based modelling that provides functional availability and support cost predictions. Implementation of this framework requires the development of an integrated hardware and service support system model. Such a model has not been found in the literature. 


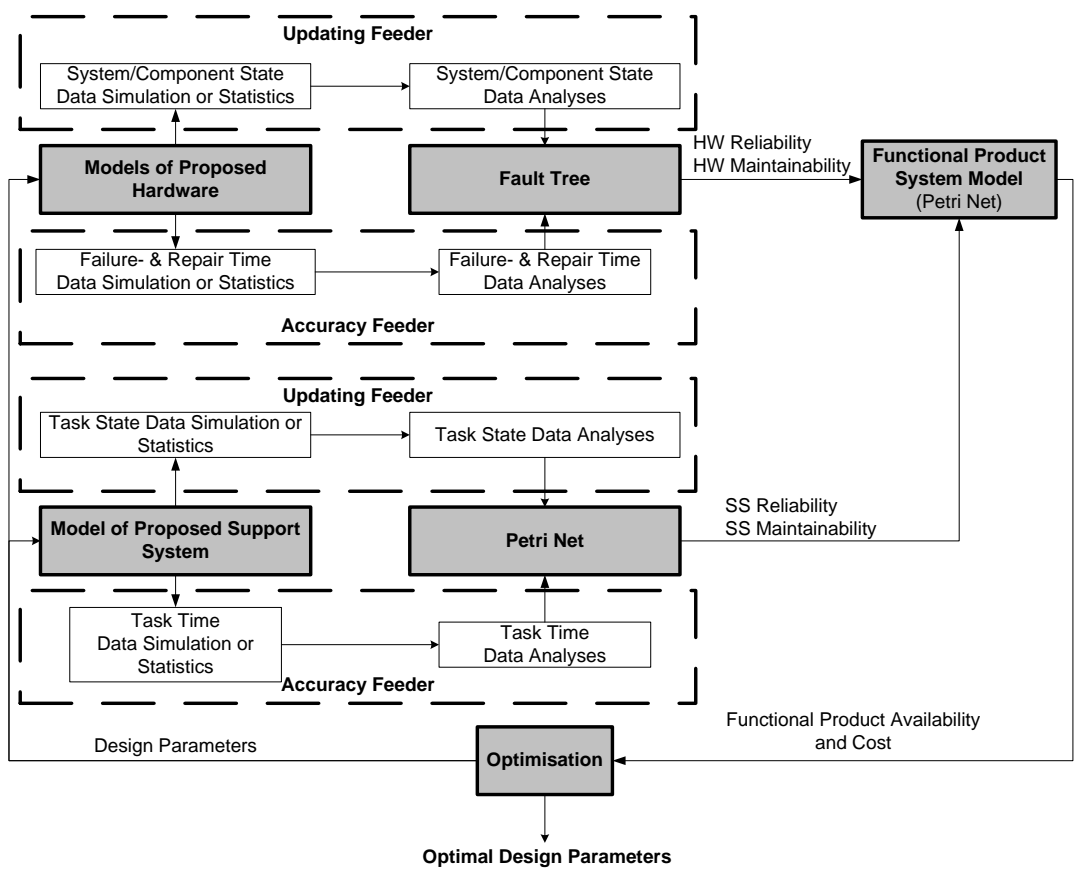

Figure 1. Schematic representation of the proposed framework (Löfstrand et al. 2011) for functional product development based on availability and cost predictions.

\section{Modelling and simulation strategy}

The literature review identified the industrial requirement for modelling tools that are able to produce hardware function availability and service support system cost predictions for functional product designs. The existence of an integrated functional product model is also essential for the implementation of the framework from Löfstrand et al (2011). In this section, an example of this type of model that has been developed and implemented by the authors is explained.

The main constituent elements of the functional product model are shown in Figure 2. Figure 2 also shows the main outputs, namely the hardware system functional availability and service support system costs. The model elements are highly integrated, mirroring the reality, and each forms part of the operational environment for the others. 


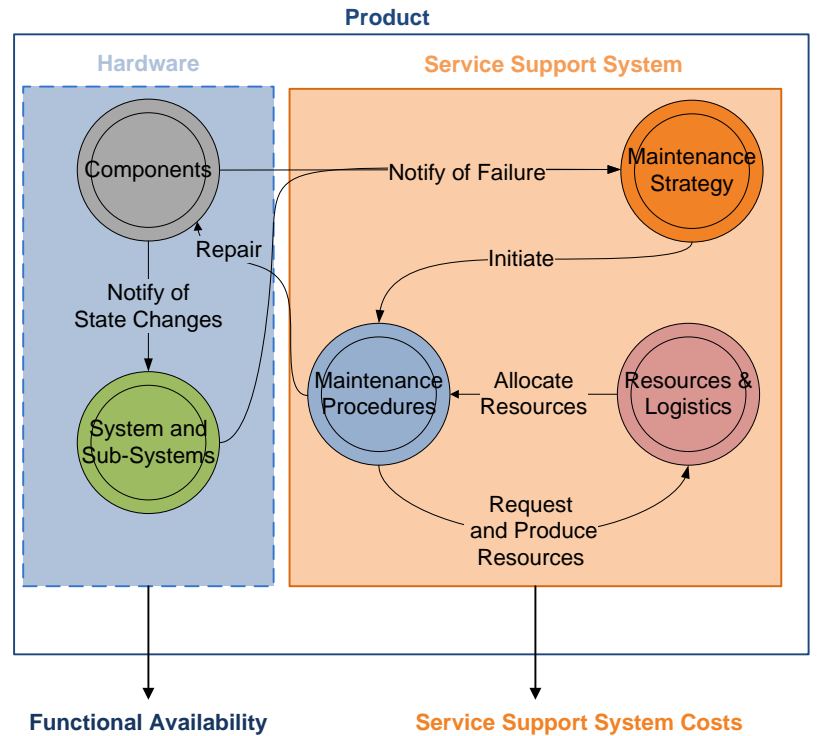

Figure 2. Constituent elements of the functional product model.

The constituent elements of the hardware shown in Figure 2 (components, systems and sub systems) correspond to the upper part of the framework schematic shown in Figure 1. The constituent elements of the service support system shown in Figure 2 (maintenance strategy, maintenance procedure, and resources and logistics) correspond to the lower part of the framework schematic shown in Figure 1. The approach for modelling each of the components from Figure 2, and their integration, will now be discussed.

\subsection{Hardware system modelling}

Functional product hardware is often complex and high-tech in nature, implying that the hardware model must be able to handle varied component failure models and complex system configurations. The modelling of hardware can be divided into the modelling of components and the modelling of subsystems, as shown in Figure 2.

\subsubsection{Component Modelling}

The set of components within a typical functional product may exhibit a variety of failure processes. Some may fail in multiple modes, whilst others may degrade in condition over a set of discrete stages. They may transition between states according to a range of different distributions. Petri nets (Schneeweiss, 1999) were therefore chosen as the technique for modelling component failure processes due to their expressive power and flexibility. A Petri net is a directed bipartite graph in which each node represents either a transition or place, shown in diagrams as a bar or hollow circle respectively. Directed arcs that link places to transitions are known as inputs, whilst those that connect transitions to places are known as outputs. Multiple input or output arcs can link the same place and transition pair, with the number of arcs known as the multiplicity. Places may contain 0 or more tokens, represented by filled circles, and it is the distribution of tokens 
Author

through the net, known as the net marking, that determines the state of the system. Each transition may be labelled with a time delay which may be fixed or determined from a random variable. Filled transitions are used when the delay time is 0 . When the number of tokens in a place matches or exceeds the number of input arcs, the transition is enabled and will fire once it has remained enabled for the duration of its associated delay, in which case the tokens are consumed from the input places, and deposited in the output places - thus altering the marking of the net and therefore the state it represents. The number of tokens consumed from the input place is equal to the number of input arcs and the number of tokens deposited in the output place is equal to the number of output arcs. If the marking of the net changes and disables a previously enabled transition, then that transition and its delay duration are reset. Only one transition can occur at any instant of time within a Petri net, regardless of the number of transitions that are enabled. Some example Petri net models for different components are shown in Figure 3. In these Petri nets, the current state of the component corresponds to the numbered place that contains a token. Appropriate distributions and parameters for the component transition times can often be found through statistical analysis of the historical times to failure or transition of similar components that have operated in a similar environment (Meeker \& Escobar, 1998). The structure of the Petri nets is such that depositing a token in a 'Repair' place returns a component to the 'as-new' state (labelled as state 1 in each of the examples). A token is deposited in a repair place by the service support system model (described in the next section) whenever a corresponding restorative maintenance procedure is completed.
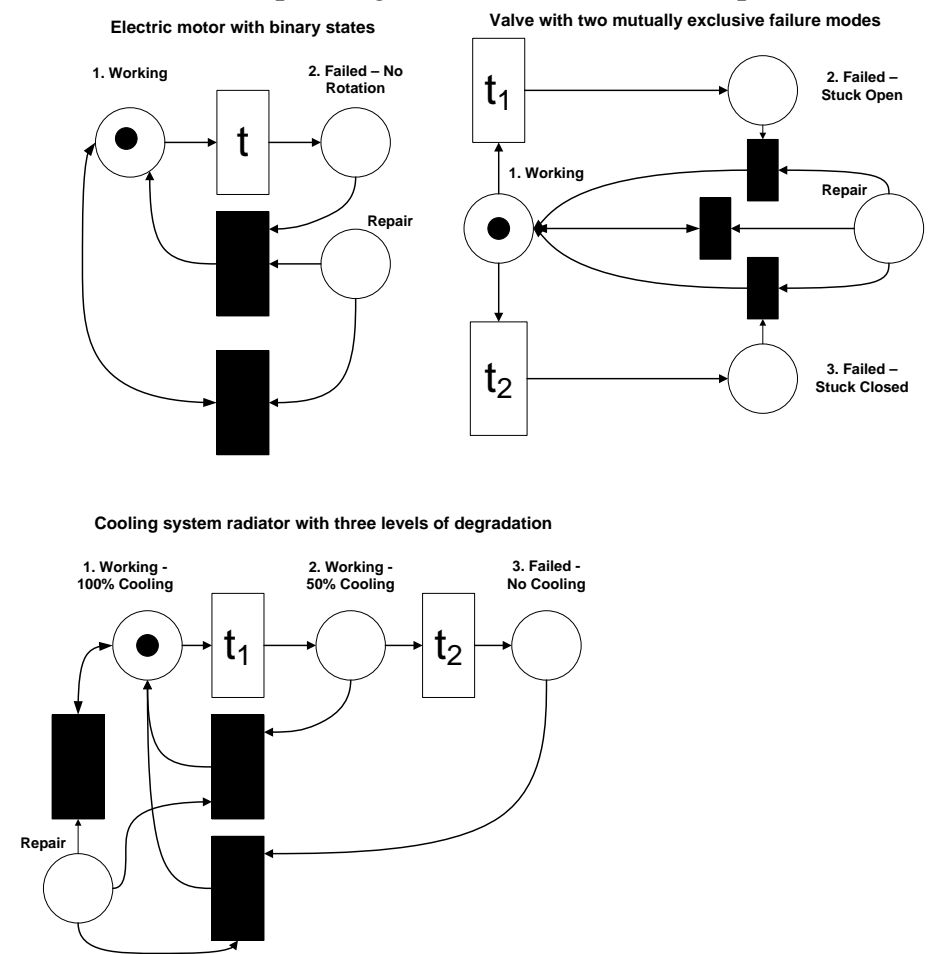

Figure 3. Petri net models representing a selection of example components. 


\subsubsection{System and Sub-System Models}

The components that form the hardware of a functional product will often to be configured such that the hardware exhibits redundancy (such that single component failures do not cause a loss of product functionality), due to the requirement for high availability. The combinations of component failures and failure modes necessary for a loss of functionality may also be complex due to the nature of the hardware design. To describe the relationship between component and functional failure within the hardware model, the fault tree technique (Andrews and Moss, 2002) is used. The top event of the fault tree represents the loss of the hardware function. This is then connected in a topdown manner via Boolean logical gates that represent intermediate events, such as subsystem failures, until basic events are reached. Basic events represent component failure, possibly in a specific failure mode or degradation condition. The state of a basic event is therefore determined from the component model Petri nets. Since a functional product provider usually supplies and supports multiple hardware items of the same or different types, the functional product model can contain many fault tree instances, each representing an individual hardware item.

\subsection{Service support system modelling}

The service support system within a functional product has a critical role in the hardware availability achieved. The service support model consists of three parts: the modelling of maintenance procedures, the modelling of maintenance resources and the modelling of maintenance strategy. Each of these will be discussed in turn in this section.

\subsubsection{Maintenance Procedures}

The method used to model maintenance procedures follows that described by Reed et al (2010) with minor modifications. Maintenance procedures are modelled as a set of tasks that are performed in a defined sequence. The completion times for tasks are modelled using distribution functions, e.g. a uniform distribution if the task has a minimum time, a maximum time and any time between those values is equally likely. Certain maintenance procedures include hardware inspection tasks and the sequence is altered dependant on the inspection outcome, for example, initiating additional maintenance tasks if a failure is found. It is also possible that a task will be performed incorrectly or will fail in some way. These failures can also alter the execution of the maintenance procedure, for example by initiating some corrective tasks or the repetition of the failed tasks. In each case, the task, whether it is an inspection or a general maintenance process, has multiple outcomes. Only tasks that are perfectly reliable, or are assumed to be perfectly reliable, and are not decision processes have a single outcome. For tasks that are based on hardware inspections, the outcome will be dependent on the state of the specified hardware component or subsystem at the point of inspection and thus determined from the hardware model. For tasks representing a general unreliable maintenance processes, each outcome is associated with a probability of occurrence such that the sum of the outcomes (for success and each possible failure mode) is unity. 
Author

The maintenance procedure sequencing constraints define, for example, which tasks must complete before another can begin. The sequence constraints are defined as follows:

- $\quad$ Each task, except the initial tasks, has one or more prerequisite sets.

- $\quad$ Each prerequisite set contains a set of inputs (i.e. the prerequisite outcomes) from other tasks (i.e. the prerequisite tasks).

- $\quad$ Once all outcomes from a prerequisite set have occurred, the task is initiated.

A maintenance procedure and its sequencing constraints can be depicted graphically through a specific type of directed graph, named an MP Graph. The graph consists of a source node, a terminal node and intermediate nodes representing the tasks. The task nodes that represent general maintenance processes are depicted by rectangles labelled with the task name, whilst those that represent hardware inspection are depicted by diamonds labelled with the component or subsystem to be inspected. Each prerequisite set for a node is represented by a set of input edges from the prerequisite tasks, labelled with the prerequisite inputs, connected to the node at the same point. An example of a MP Graph is shown in Figure 4. In this maintenance procedure, task 1 is performed first followed by task 2 if completed successfully (with a probability of 0.8 ) or by task 3 if it fails (with a probability of 0.2 ). On completion of task 2 or task 3 , an inspection occurs and the procedure ends if component $\mathrm{A}$ is not failed otherwise task 4 is initiated and the procedure ends on its completion.

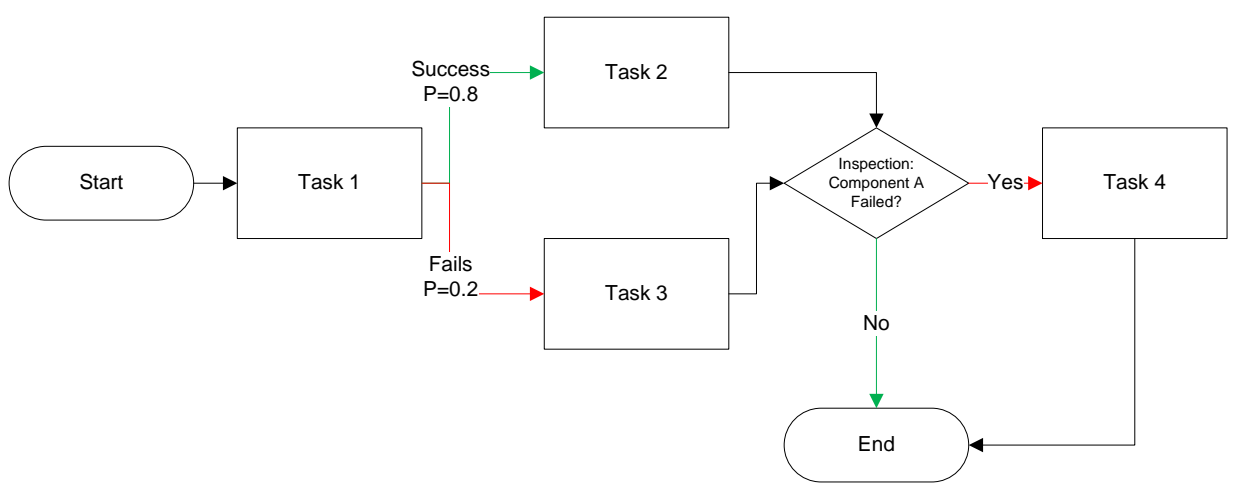

Figure 4. An example of an MP Graph.

Maintenance procedures are input to the software through descriptions of their MP Graph structure, but the modelling implementation converts them into Timed Petri net representations prior to simulation. Timed Petri nets are ideal for representing systems that exhibit concurrent, sequential and competitive activities (Wang, 1998). Each general maintenance process task is converted into a Petri net of the form shown in Figure 5. As depicted, the Petri net interacts with the resource model which is described in chapter 3.2.2 below. The Petri net representation of a hardware inspection is shown in Figure 6. 


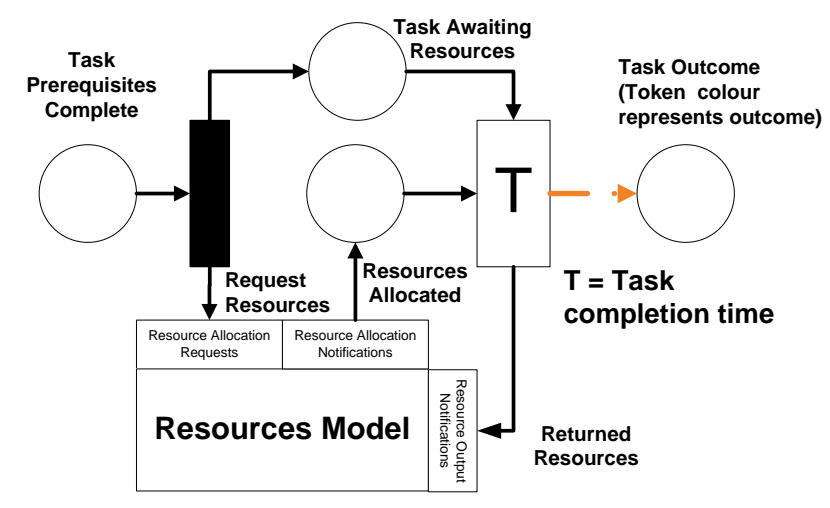

Figure 5. Petri net representation of a general maintenance process task.

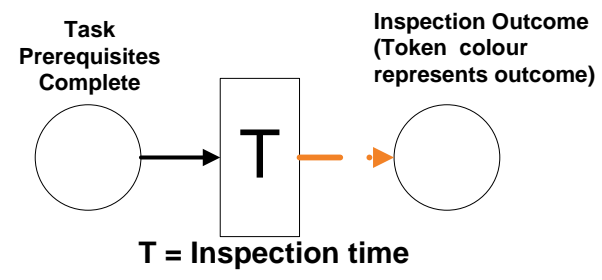

Figure 6. Petri net representation of an inspection maintenance task.

The task Petri nets are integrated together to form a Petri net representation of an overall maintenance activity from which the total completion time for the maintenance activity can be found. Figure 7 shows the Petri net representing the example maintenance procedure shown in Figure 5, where the transitions labelled $T_{1}, T_{2}, T_{3}$ and $T_{4}$ represent the Petri nets for tasks 1 to 4 of the form shown in Figure 5 ; the transition labelled $T_{i}$ represents the Petri net for the inspection of component $A$ of the form shown in Figure 6; the dashed output edges are labelled with the possible outcome token colours; the filled transitions are labelled with the enabling token colour where applicable. 


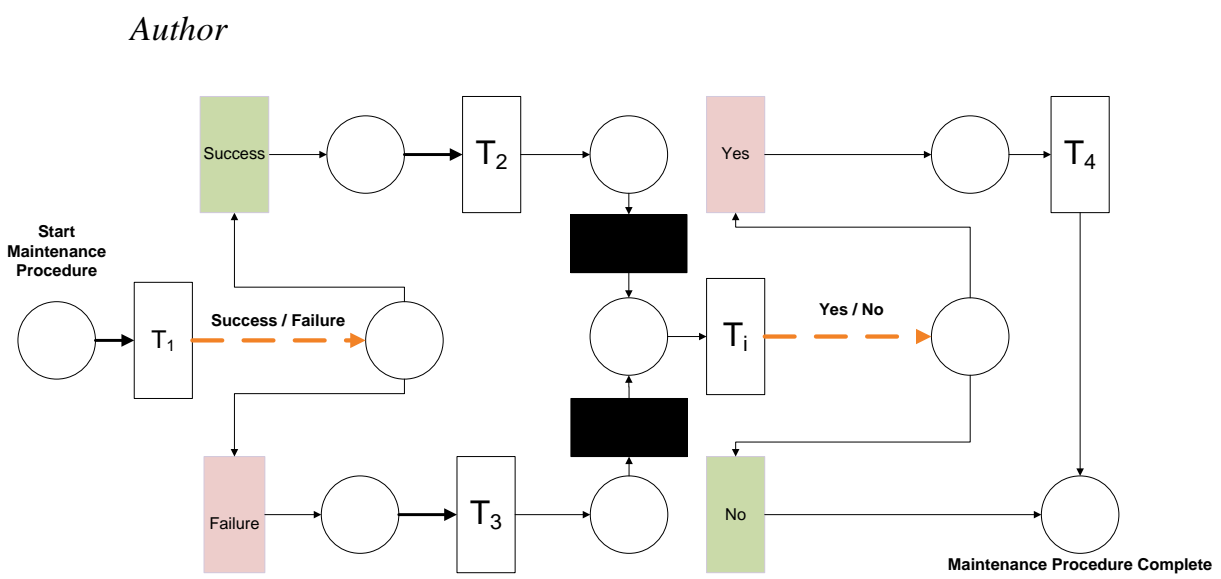

Figure 7. Petri net representation of the example maintenance procedure shown in Figure 5.

\subsubsection{Maintenance Resources}

Maintenance tasks require resources to perform, including spare parts, facilities, tools and personnel. A maintenance task may consume resources (e.g. spares), utilise resources for its duration (e.g. personnel, tools) and produce resources upon completion (e.g. overhauled parts). To model this, each maintenance task is associated with its maintenance resource requirements. The utilisation of resources over a sequence of tasks, or over a complete maintenance procedure, is modelled by associating the initial task in the sequence as a consumer of the resources and associating the final task in the sequence as a producer of the resources. The availability of resources will depend on the tasks currently in-progress and the overall resource levels, which may be replenished when orders arrive from suppliers. The provision of maintenance resources is also a primary cost within service support systems. For these reasons, accurate modelling of maintenance resource availability is important within a functional product model. The different processes that might dictate overall maintenance resource availability are wide ranging and complex, and must therefore be implemented through customised logic on a case by case basis. Those that might be modelled include the purchasing of resources from suppliers, transport of inventory between repair depots and shift patterns for the availability of personnel. The interface between the maintenance model and resource model has been generalised however, allowing them to be developed in an independent and modular manner. Once a task is ready to begin it creates a demand for the resources it requires, as shown in Figure 5. The resource model then allocates the requested resources, either as soon as they become available or based on customised allocation logic. On completion of a task, the maintenance model notifies the resource model, which then updates the resource levels with those that were freed from utilisation or produced.

\subsubsection{Maintenance Strategy}

The maintenance strategy consists of the set of maintenance policies that determine when maintenance procedures are initiated within the service support system. The possible maintenance policies for individual hardware items include: 


\section{Modelling and Simulation of Functional Product System Availability}

- Corrective - for items with revealed failure, a corrective maintenance policy causes maintenance to be initiated as soon as the item fails.

- Periodic inspection - for items with unrevealed failure, a scheduled inspection policy results in an inspection that is carried out periodically. Maintenance is then initiated if the item is failed when that inspection is carried out.

- Periodic restoration - A periodic preventive maintenance policy causes maintenance to be initiated periodically, regardless of the state of the item.

A manufacturer may want to use any combination of these to maximise the performance of a functional product. The maintenance strategy is modelled by allowing any number of maintenance policies to be created, of any of the types above and with the relevant parameters set (e.g. the periodic inspection period), where each policy is associated with a particular maintenance procedure and set of hardware items. Whenever the policy is triggered, the associated maintenance procedure begins, and the associated hardware items are restored to the 'as-new' condition upon completion. The triggering of a policy is dependent on its type, for example, a corrective policy is triggered when the associated hardware items enter a failed state. This enables the modelling of most maintenance strategies that might be encountered in practice. The maintenance strategy model provides the link that integrates the hardware and service support system models.

\subsection{Model Simulation}

Discrete event simulation (DES) (Banks, 1998) can be used to simulate the model described in the previous sections and evaluate the performance of a functional product over a certain period of operation. DES is a powerful method for analysing models containing random processes where events, such as component failures and maintenance task completions, occur at specific points in time. A simulation involves the generation of a large number of trials, each representing an artificial history for the functional product over an operating period, and from that collection of histories the statistical behaviour, characteristics and performance of the system can be calculated. Analytical methods are not suitable as the complexity of the functional product model is too great and simplifications or assumptions would need to be made that would severely limit its usefulness. An added benefit is that DES can generate very detailed output data that may be used to evaluate the performance of a functional product or as input data to an optimisation process, as described in the framework outline. The expected uptime of the functionality for a single hardware item over a simulated time period $T, E(U)$, can be calculated over $n$ simulation trials through Equation 1, where $X_{i}(t)$ is the status of the hardware function at time $t$ in trial $i$ as given by Equation 5. Similarly, the expected downtime is given by Equation 2. The mean availability for the functionality of the hardware item over time period $T$ is then given by Equation 3 . The availability at time $t$, $A(t)$, is defined as the probability that the hardware item functionality is available at time $t$ and can be calculated as shown in Equation 4 .

$$
E(U)=\frac{1}{n} \sum_{i=1}^{n} \int_{0}^{T} X_{i}(t) d t
$$


Author

$$
\begin{gathered}
E(D)=\frac{1}{n} \sum_{i=1}^{n} \int_{0}^{T} 1-X_{i}(t) d t=T-E(U) \\
A=\frac{E(U)}{E(U)+E(D)} \\
A(t)=\operatorname{Pr}(X(t)=1)=E(X(t))=\frac{1}{n} \sum_{i=1}^{n} X_{i}(t) \\
X_{i}(t)= \begin{cases}1, & \text { hardware function available at time } t \\
0, & \text { otherwise }\end{cases}
\end{gathered}
$$

The generation of a simulation trial will now be explained. During the simulation trials, events are generated from the various parts of the model. The number of types of event that are generated is too great for each to be mentioned separately; however an example is given below for illustrative purposes:

When a multistate component is new, is repaired or enters a new state, an event for its next transition is generated. This event is set to occur after a waiting time equal to the transition time and when executed causes the component to enter a new state. The transition time and arrival state are found by first generating times for each possible transition from the current state, with each time found by taking a random sample from the associated transition time distribution. The smallest transition time value and associated arrival state is then selected as the transition that occurs.

The occurrence of an event often causes other events to be generated. For example, when a repair event occurs, returning a component to the 'as new' state, a new failure event is generated. When a failure event for a component with a corrective maintenance policy occurs, a maintenance procedure event will be generated, which itself may cause the generation of numerous task completion events. A trial ends when the simulated operating time for the functional product is reached. Figure 8 is a flowchart showing the DES process where tmax is the operating period for the functional product to be modelled. Simulating a greater number of trials results in better accuracy at the expense of increased computation time. 


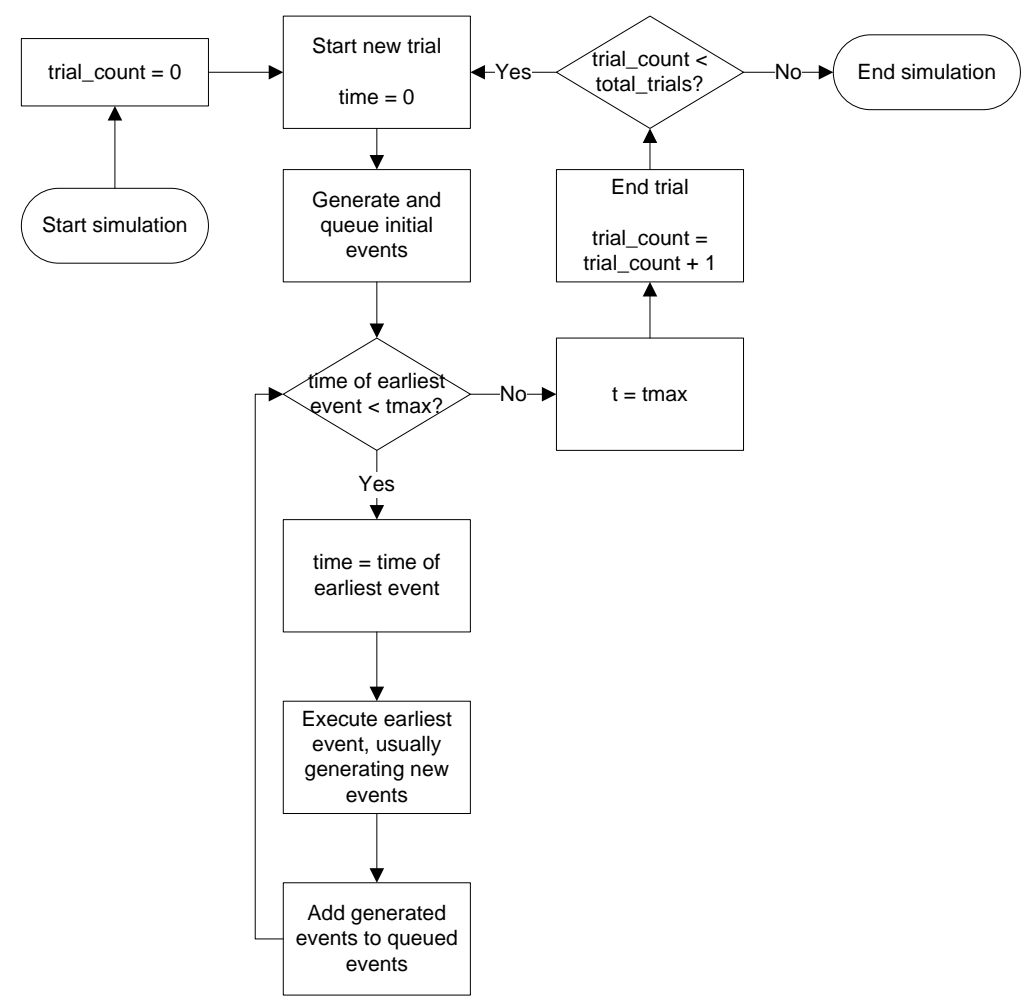

Figure 8. Flowchart of Discrete Event Simulation Process.

\subsection{Model Implementation}

The model described in the previous sections has been implemented as a software tool written in the C\# programming language. The inputs to this tool in order to model a specific functional product include: the component descriptions, the hardware system fault trees, the maintenance task descriptions, the maintenance procedure descriptions and a resource model. This input data is input in XML format (Bray et al. 1999) and the included information can be obtained by a manufacturer during product development. Additional input parameters for the simulation settings include: the operating period to be simulated, the number of trials to be simulated and the model parameters to monitor (for example, the hardware system state might be monitored in order to later calculate its availability).

Once the tool has simulated a functional product, the data collected from the monitored model parameters can be analysed to produce useful metrics. These metrics include the:

- $\quad$ Expected hardware availability 
Author

- Variance in hardware availability

- Mean maintenance service times

- Maximum maintenance service times

Detailed output data, such as plots of the reliability of a service against time, can also be produced.

The main elements of the functional product model and its implementation are shown in Figure 9.

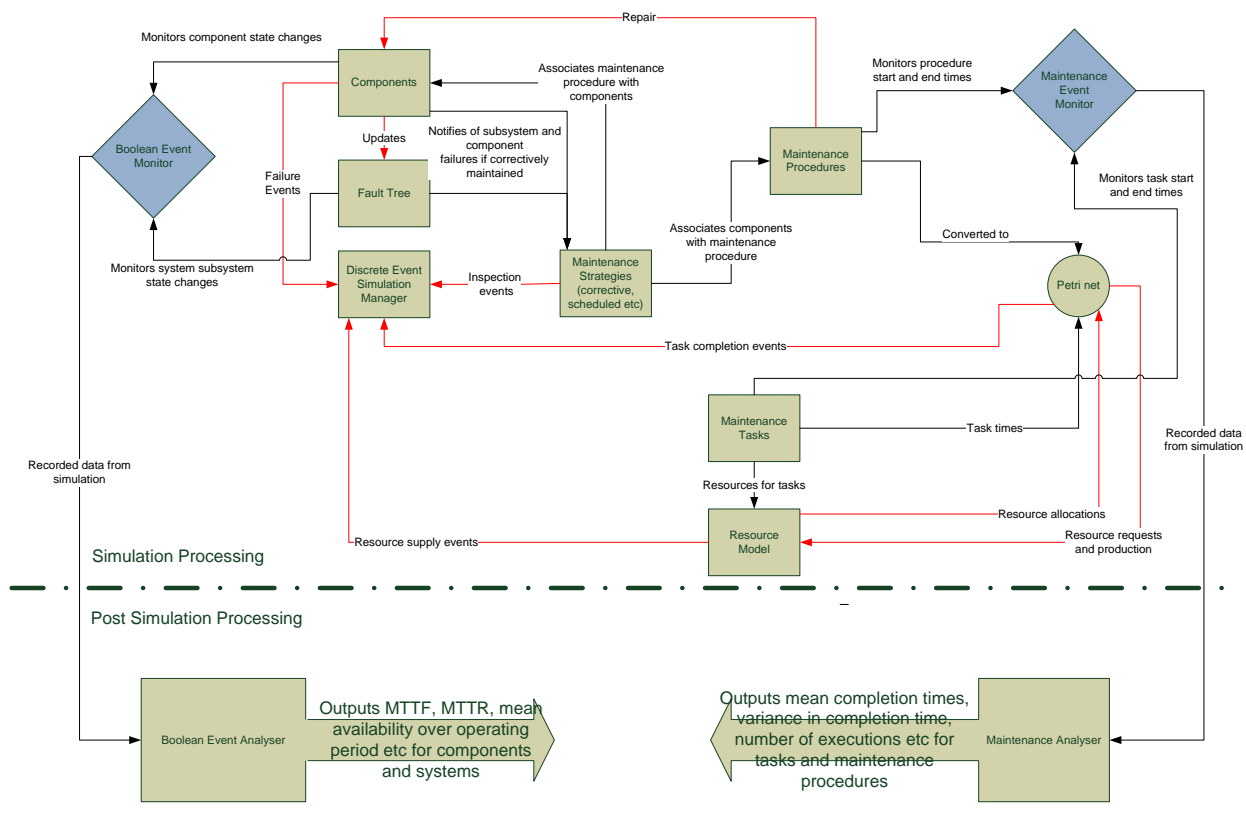

Figure 9. Overview of the main model elements and its implementation.

\section{A verifying example}

The developed model and its implementation are demonstrated in this section by applying it to the analysis of a simple example functional product. Note that neither the model nor implementation is limited to the analysis of simple examples of the type exemplified here. It can be similarly applied to more typical real world scenarios involving the support of multiple complex functional product hardware items.

The hardware system consists of four components, named $A, B, C$ and $D$. Components $A, B$ and $C$ are binary state components that are either working or failed at any time (see the electric motor example in Figure 3). Loss of availability of the functional product occurs when subsystem 1 is failed, which occurs if $A$ and $C$ are simultaneously failed, or when component $B$ is failed, as shown by the fault tree in Figure 10. Component $D$ is a multi-state component that provides cooling for component $B$ and 


\section{Modelling and Simulation of Functional Product System Availability}

its performance degrades with age in discrete steps, as shown by the Petri net in Figure 11 . The hazard rate for component $B$ increases with the degradation of component $D$. The proportional hazard factor for component $B$ which gives the multiple of its underlying hazard function, i.e. its hazard function when component $D$ has no degradation, is shown in Table 1. Thus, although it does not appear in the fault tree, the maintenance of component $D$ influences the hardware availability through its influence on component $B$ which does appear in the fault tree. The failure distributions for components $A, B$ and $C$ are given in Table 2, where component $C$ has an exponential distribution (constant failure rate) and components $A$ and $B$ have Weibull distributions. Note that the distribution given for component $B$ is its underlying failure distribution when component $D$ is functioning perfectly, thus the actual failure rate for component $B$ is given by Equation (1) where $\phi$ is the proportional hazard factor whose values are given in Table 1.

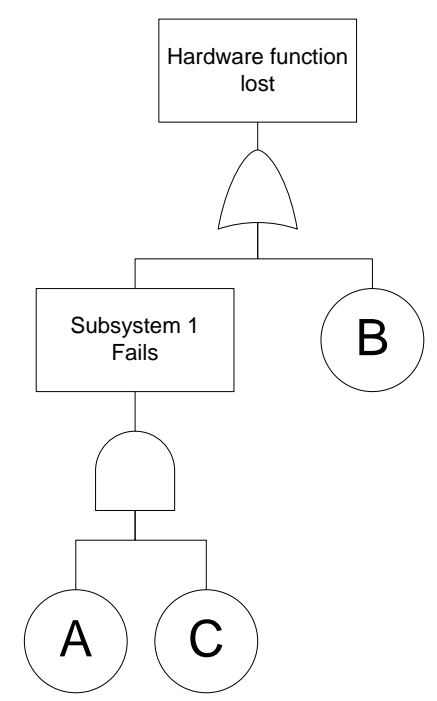

Figure 10. Fault tree, representing functional failure, for example functional product. 
Author

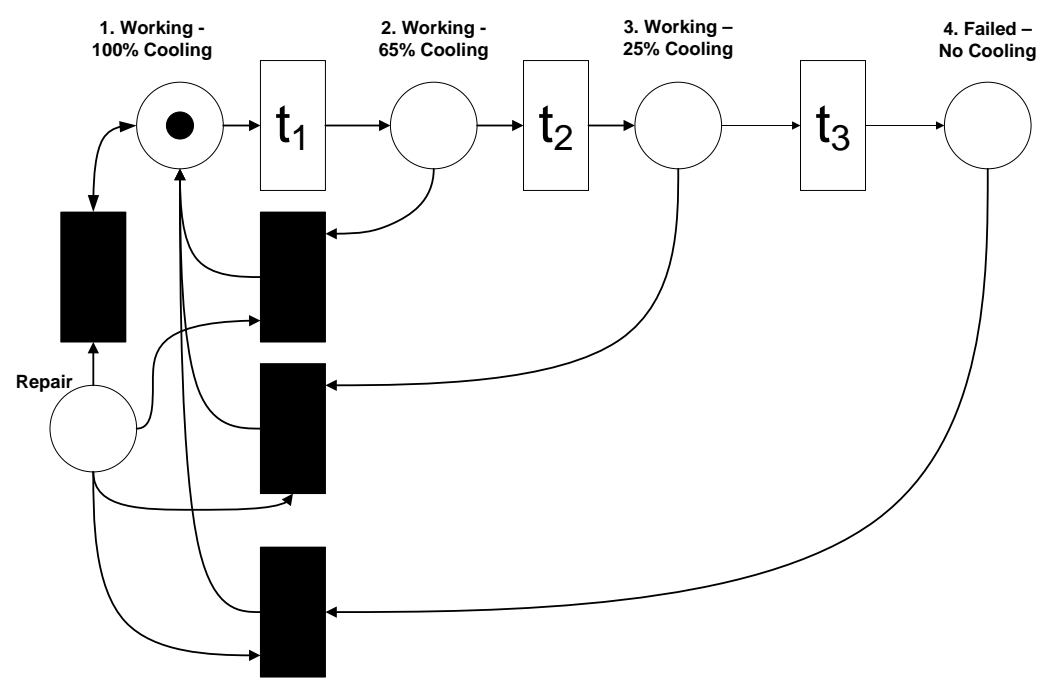

Figure 11. Petri net for component D.

Table 1. Proportional hazard factors.

\begin{tabular}{|l|l|}
\hline State of component D & $\begin{array}{l}\text { Proportional Hazard Factor } \boldsymbol{\phi} \text { for } \\
\text { Component B }\end{array}$ \\
\hline Working - 100\% Cooling & 1 \\
\hline Working - 65\% Cooling & 1.5 \\
\hline Working - 25\% Cooling & 2 \\
\hline Failed - 0\% Cooling & 2.7 \\
\hline
\end{tabular}

Table 2. Failure distributions.

\begin{tabular}{|l|c|}
\hline Component & Failure Distribution \\
\hline A & $F(t)=1-e^{-\left(\frac{t}{1500}\right)^{3}}$ \\
\hline B & $F(t)=1-e^{-\left(\frac{t}{3000}\right)^{2.2}}$ \\
\hline C & $F(t)=1-e^{-\frac{t}{2000}}$ \\
\hline
\end{tabular}


Modelling and Simulation of Functional Product System Availability

$$
h(t)=\phi \frac{2.2}{3000}\left(\frac{t}{3000}\right)^{1.2}
$$

The transition rates for component $\mathrm{D}$ are given in Table 3.

Table 3. Transition rates for component $D$.

\begin{tabular}{|l|l|}
\hline Transition & Rate \\
\hline $100 \%$ to $65 \%$ Cooling & 0.004 \\
\hline $65 \%$ to $25 \%$ Cooling & 0.005 \\
\hline $25 \%$ to $0 \%$ Cooling & 0.005 \\
\hline
\end{tabular}

Components $A$ and $C$ have unrevealed failures, but the working or failed status for component $C$ can be found through inspection. However, due to its location within subsystem 1 , it is only practical to inspect component $C$ during the replacement of component $A$. The failure of subsystem 1 reveals that component $A$ and $C$ have failed, as can be determined from the fault tree shown in Figure 10. Failures of component $B$ are revealed and its status at any time is therefore known. Failure of component $D$ are also revealed, however its degradation whilst in the working state cannot be determined. Repair or replacement of any of the components returns the repaired components to the 'as-new', or perfect, condition.

The available maintenance procedures, named I to IV, are shown in Figure 12 to Figure 15. Maintenance procedure I, from Figure 12, replaces component $A$ and also component $C$ if it is found to be failed at the point of inspection. Maintenance procedure II, from Figure 13 replaces both components $A$ and $C$. Finally, maintenance procedures III and IV, shown in Figure 14 and Figure 15 respectively repair component $B$ and component $D$ respectively. Each maintenance procedure is associated with a cost as follows: 3 for maintenance procedure $I, 2$ for maintenance procedure II and 1 for maintenance procedures III and IV.

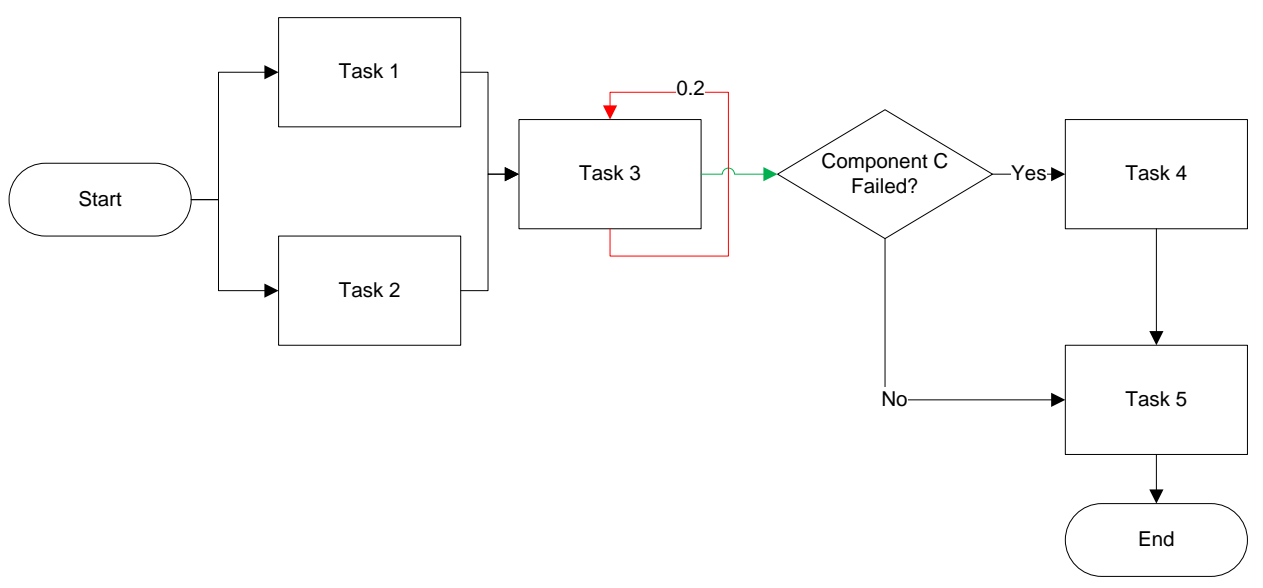

Figure 12. Maintenance Procedure I. 


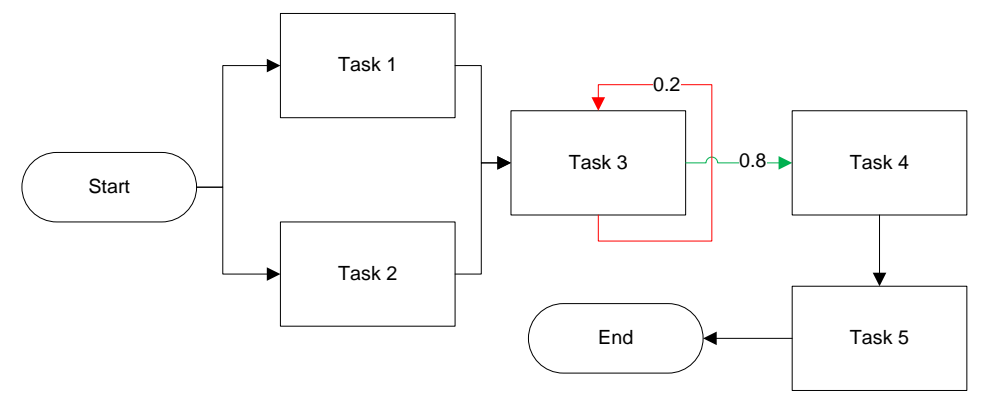

Figure 13. Maintenance Procedure II.

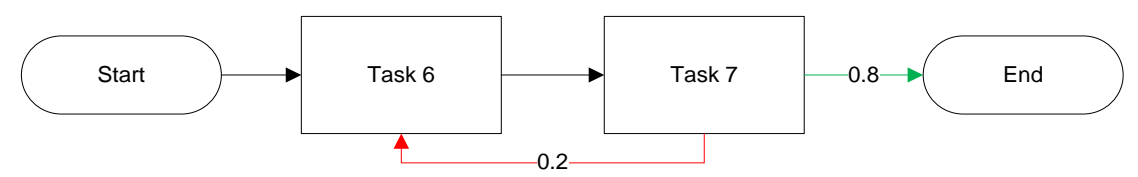

Figure 14. Maintenance Procedure III.

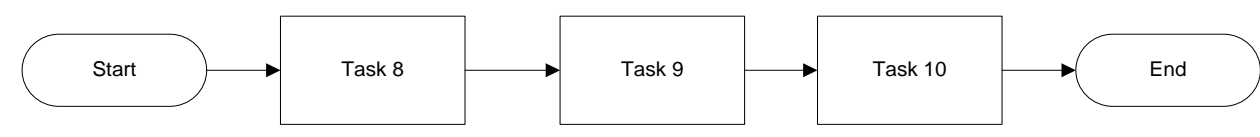

Figure 15. Maintenance Procedure IV.

The completion times for all tasks from the maintenance procedures are assumed to be uniformly distributed with the minimum and maximum completions times indicated in Table 4. Each task utilises a single maintenance engineer during its completion and engineers can switch freely between procedures on completion of a task - thus an engineer can, for example, complete a task from one procedure, complete a task from another procedure and then return to the original procedure (i.e. they are not required to remain occupied by a single maintenance procedure for its duration). 
Modelling and Simulation of Functional Product System Availability

Table 4. Maintenance procedure task data.

\begin{tabular}{|l|l|l|l|}
\hline Task Number & $\begin{array}{l}\text { Minimum } \\
\text { Completion } \\
\text { (hrs) }\end{array}$ & $\begin{array}{l}\text { Maximum } \\
\text { Completion } \\
\text { (hrs) }\end{array} \quad$ Time & Reliability \\
\hline 1 & 5 & 10 & 1 \\
\hline 2 & 3 & 5 & 1 \\
\hline 3 & 6 & 12 & 0.8 \\
\hline 4 & 2 & 4 & 1 \\
\hline 5 & 1 & 2 & 1 \\
\hline 6 & 4 & 6 & 1 \\
\hline 7 & 2 & 6 & 0.8 \\
\hline 8 & 4 & 8 & 1 \\
\hline 9 & 2 & 4 & 1 \\
\hline 10 & 2 & 4 & 1 \\
\hline
\end{tabular}

The functional product model was simulated over a period representing 2 years $(17,520 \mathrm{hrs})$ of operation. The predicted performance of the functional product was evaluated for two different maintenance strategies, named $A$ and $B$, with 10,000 trials simulated for each.

Maintenance strategy $A$ is as follows:

- Maintenance procedure I is performed every $500 \mathrm{hrs}$.

- Maintenance procedure III is performed on failure of component B.

- Maintenance procedure IV is performed every $400 \mathrm{hrs}$.

- 2 maintenance engineers are available, at a total cost of 100 over the two year period.

Maintenance strategy B is as follows:

- Maintenance procedure II is performed on failure of subsystem 1.

- Maintenance procedure III is performed on failure of component B.

- Maintenance procedure IV is performed on failure of component D.

- 1 maintenance engineer is available, at a cost of 50 over the two year period.

\subsection{Example outputs and results}

Under maintenance strategy $A$ the expected uptime (Equation 1) over the two year period was 17,041 hrs, giving availability (Equation 3) of $97.3 \%$. Under maintenance strategy $B$, the expected uptime over the two year period was just over 200 hrs greater at 17,254 hrs, resulting in an availability of $98.3 \%$. Figure 16 shows a plot of availability (Equation 4) against time for the hardware system under the two different maintenance strategies. The plot shows that that the availability level is also far more consistent over the two year period with maintenance strategy $B$, whereas under strategy $A$ it has relatively higher variability, with low availability prior to scheduled maintenance and high availability for a short time afterwards. The plot also shows that the initial availability at time 0 is 1 and reaches the steady state after around 2500hrs. The expected 
costs incurred due to execution of maintenance procedures under each strategy are shown in Table 5. This shows that the expected costs of implementing maintenance strategy $A$ are almost twice as great as for strategy $B$.

The managerial implications of the simulation analysis would be to conclude that strategy $B$ is preferable to strategy $A$ for the manufacturer, since it results in both better availability and lower support costs. An objective function can be used to evaluate different product designs in terms of all relevant factors in more complex scenarios.

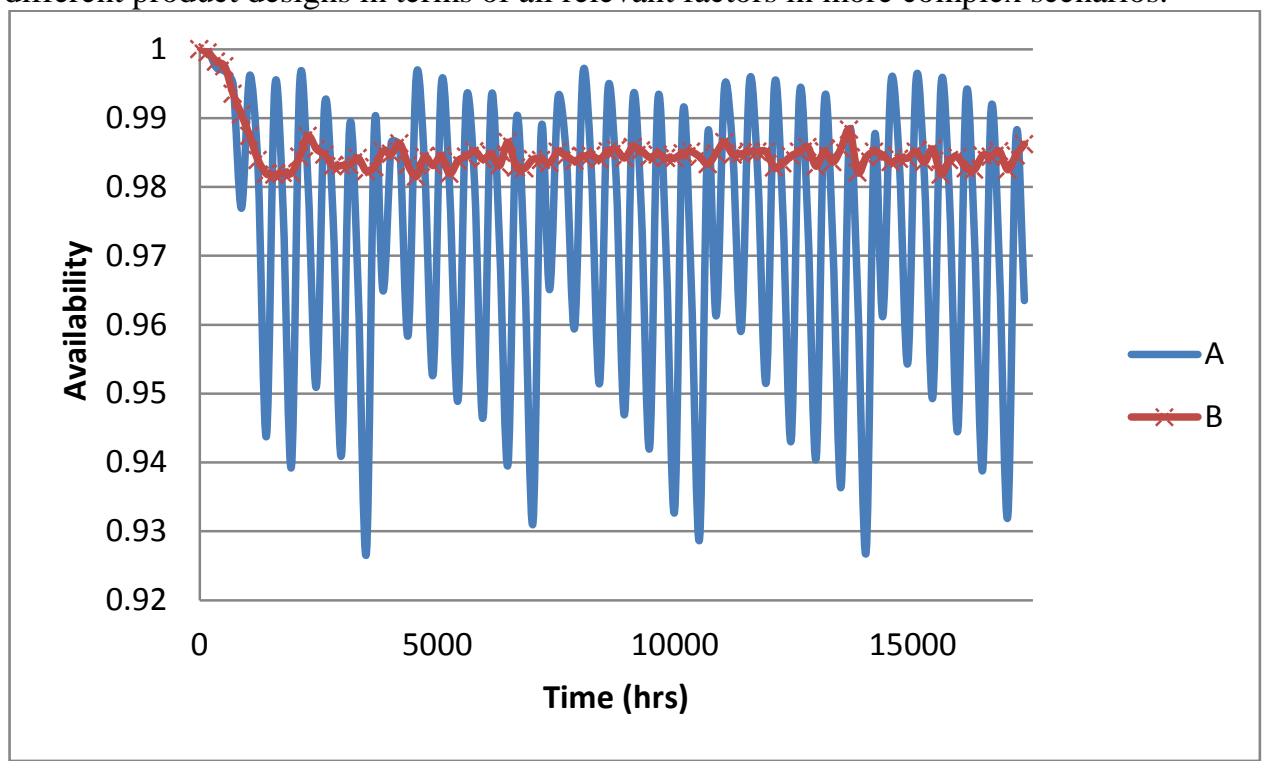

Figure 16. Plot of availability against time for hardware system under maintenance strategies A and B.

Table 5. Expected cost incurred from executing maintenance procedures under each maintenance strategy.

\begin{tabular}{|l|l|l|}
\hline Maintenance Procedure & Expected Cost Strategy A & Expected Cost Strategy B \\
\hline I & $105.00(35$ times $)$ & 0 \\
\hline II & 0 & 13.98 \\
\hline III & 7.24 & 6.27 \\
\hline IV & 43.00 & 66.82 \\
\hline $\begin{array}{l}\text { Total (including engineer } \\
\text { costs) }\end{array}$ & $155.24(255.25)$ & $87.07(137.07)$ \\
\hline
\end{tabular}

Analysis such as that shown in this example fits into the framework model, where the optimisation loop output would alter the value of variables, such as the component replacement intervals, and receive as input the metrics used to evaluate the performance of the functional product, such as hardware availability and mean service support system response times. 
Modelling and Simulation of Functional Product System Availability

\section{Summary and Conclusions}

Manufacturers are increasingly looking to offer functional products due to the benefits they offer to both themselves and customers. The research literature makes it clear that there is a need for tools that can support manufacturers in developing and offering these products, particularly those that can assess the value of particular product design decisions or designs. There is already a wealth of methods, and tools that utilise them, for analysing hardware reliability. More recently, methods for analysing service support systems have been developed (Li \& G. Thompson 2007, Reed et al. 2010). Löfstrand et. al. (2011) developed a framework that contains an outline of how a simulation based functional product model, integrating both hardware and service support system models, will enable the prediction of hardware functional availability and service support system costs. A methodology that implements that part of the framework has been outlined in this paper. This methodology uses a combination of discrete event simulation, Petri net and fault tree analysis and is suitable for modelling the types of complex, high-tech products that are best suited for functional product offers.

The presented methodology has been implemented as a software tool. The input data for this tool can be obtained by a manufacturer during product development. This software tool has been demonstrated through application to a simple example of a functional product. In this example, the product functional availability was analysed under two different maintenance strategies. The output showed a clear advantage, in terms of hardware availability and support costs, when one of those was used in comparison to the other. This shows how the tool might be used by a functional product manufacturer to compare functional product design decisions and evaluate the performance of particular designs. Future development of the methodology and implementation will include the addition of update and optimisation loops, as envisioned in the framework by Löfstrand et. al. (2011).

\section{Acknowledgments}

This work was partly conducted at the Faste Laboratory, Luleå University of Technology, a VINNOVA (The Swedish Governmental Agency for Innovation Systems) Excellence Center.

John Andrews is the Royal Academy of Engineering and Network Rail Professor of Infrastructure Asset Management. He is also Director of The Lloyd's Register Educational Trust $^{1}$ Centre for Risk and Reliability Engineering at the University of Nottingham.

\footnotetext{
${ }^{1}$ The Lloyd's Register Educational Trust (The LRET) is an independent charity working to achieve advances in transportation, science, engineering and technology education, training and research worldwide for the benefit of all.
} 
Author

\section{References}

Alonso-Rasgado, T., Thompson, Graham \& Elfström, B.-O., 2004. The design of functional (total care) products. Journal of Engineering Design, 15(6), pp.515-540.

Andrews, J, 2009. System reliability modelling: the current capability and potential future developments. Proceedings of the Institution of Mechanical Engineers, Part C: Journal of Mechanical Engineering Science, 223(12), pp.2881-2897. Available at: http://pic.sagepub.com/lookup/doi/10.1243/09544062JMES1538 [Accessed December 12, 2011].

Andrews, J.D. \& Moss, B., 2002. Reliability and Risk Assessment 2nd ed., Wiley-Blackwell.

Baines, T.S. et al., 2007. State-of-the-art in product-service systems. Proceedings of the Institution of Mechanical Engineers, Part B: Journal of Engineering Manufacture, 221(10), pp.15431552. Available at: http://journals.pepublishing.com/openurl.asp?genre=article\&id=doi:10.1243/09544054JEM8 58 [Accessed June 10, 2011].

Banks, J., 1998. Handbook of Simulation: Modelling, Estimation and Control, John Wiley \& Sons.

Bray, T., Paoli, J. \& Sperberg-McQueen, C.M., 1999. Extensible Markup Language (XML) 1.0,

Brännström, O., Elfström, B.-O. \& Thompson, Graham, 2001. Functional Products Create New Demands on Product Development Organisations. International Conference on Engineering Design.

Kim, S.-H., Cohen, M. a \& Netessine, S., 2007. Performance Contracting in After-Sales Service Supply Chains. Management Science, 53(12), pp.1843-1858. Available at: http://mansci.journal.informs.org/cgi/doi/10.1287/mnsc.1070.0741.

Li, J.-p \& Thompson, G., 2007. Simulation-based Reliability Assessment of Services in the Context of Functional Products,

Löfstrand, M. et al., 2011. Functional product system availability: simulation-driven design and operation through coupled multi-objective optimisation. International Journal of Product Development, 13(2), pp.119-131.

Meeker, W.Q. \& Escobar, L.A., 1998. Statistical Methods for Reliability Data, Wiley-Blackwell.

Reed, S. et al., 2010. Modelling Service Support System Reliability. In IFAC A-MEST. Lisbon: IFAC. 